\title{
Current Possible Drug Therapies for Ovarian Cancer
}

\author{
Jungyeob Seoung, Young-Han Park*, Chaechun Rhim, Sungju Kim \\ Division of Gynecologic Oncology, Department of Obstetrics and Gynecology, Hallym University Sacred Heart \\ Hospital, Anyang, South Korea \\ Email: "drparkyh@yahoo.co.kr
}

Received 21 August 2014; revised 18 September 2014; accepted 15 October 2014

Copyright $@ 2014$ by authors and Scientific Research Publishing Inc.

This work is licensed under the Creative Commons Attribution International License (CC BY).

http://creativecommons.org/licenses/by/4.0/

(c) (7) Open Access

\begin{abstract}
Ovarian cancer is the most aggressive gynecologic cancer of the heterogenous phenotypes. Development of the new chemotherapeutic agents and drug delivery mode makes better outcomes for patient treatments. Optimal cytoreductive therapy followed by molecular targeting therapy, intraperitoneal chemotherapy and dose dense chemotherapy is a hot therapeutic concept in ovarian cancers. In our review, we will introduce recent therapeutic advances in epithelial ovarian cancer patients.
\end{abstract}

Keywords

Ovarian Cancer, Intraperitoneal Chemotherapy, Targeting Therapy

\section{Introduction}

Epithelial ovarian cancer (EOC) is the most lethal malignancy in gynecologic cancer. It is estimated that 22,240 cases were diagnosed with EOC and 14,030 deaths from EOC occurred in the US in 2013. The cure rate of this disease is less than $40 \%$ [1]. At the time of diagnosis, most patients have the advanced stage disease. The standard treatment of newly diagnosed EOC is the optimal cytoreductive surgery and followed platinum-based chemotherapy. Despite advances in surgical and chemotherapeutic strategies, the majority will experience disease recurrence requiring further treatment. Over $70 \%$ of the patients with advanced stage EOC experience recurrence within 2 years after initial treatment.

Resistance to chemotherapy is a major clinical problem. Multiple signaling pathways have been implicated in the resistance to chemotherapy. To overcome the resistance to therapy, innovative therapeutic strategies are needed. In this review, we will summarize current treatment strategy with conventional cytotoxic agents includ-

${ }^{*}$ Corresponding author. 
ing intraperitoneal (IP) chemotherapy and dose dense chemotherapy schedule. We also focused our attention on reviewing current phase 3 trials with promising target agents to overcome conventional cytotoxic agents and improve outcome of EOC (Table 1).

\section{Cytotoxic Chemotherapy}

\subsection{Finding Optimal Regimen of Conventional Cytotoxic Agent; Dose Dense Chemotherapy}

Currently, the combination of paclitaxel and carboplatin is the standard chemotherapy regimen for EOC. This consists $175 \mathrm{mg} / \mathrm{m}^{2}$ of paclitaxel given intravenously over 3 hours, followed by carboplatin intravenous (IV) infusion over 30 - 60 min at a dose of AUC 5 - 6. This is repeated every 3 weeks for six cycles. Dose dense therapy is strategy to enhance antitumor activity to focus on rapid regrowth of tumor between treatment cycles. Increasing the dose density of chemotherapy is expected to increase efficacy by minimizing the opportunity for regrowth of tumor cells [2]. The Japanese Gynecologic Oncology Group (JGOG) conducted a randomized phase 3 trial of dose-dense weekly paclitaxel in combination with 3-weekly carboplatin for advanced EOC (JGOG 3016, NOVEL trial) [3]. In this study, Median PFS was longer in the dose-dense treatment group (28.0 months, 95\% CI 22.3 - 35.4) than in the conventional treatment group (17.2 months, 15.7 - 21.1; HR0.71; 95\% CI 0.58 0.88 ; $\mathrm{p}=0.0015)$. Overall survival (OS) at 3 years was higher in the dose-dense regimen group $(72.1 \%)$ than in the conventional treatment group (65.1\%; HR 0.75, $0.57-0.98$; $p=0.03)$. Patient treated with dose dense therapy had a significantly higher frequency of hematologic toxicity, and a lower treatment completion rate ( $63 \%$ vs. 48\%). In long term follow-up data of this trial, median PFS was significantly longer in the dose-dense treatment group than in the conventional treatment group (28.2 months vs. 17.5 months, HR 0.76, 95\% CI $0.62-0.91, \mathrm{p}=$ 0.0037). Median OS was 100.5 months in the dose-dense treatment group and 62.2 months in the conventional treatment group (HR 0.79, 95\% CI 0.63 - 0.99, p = 0.039) [4].

Confirmatory studies are ongoing in western countries. In the United States, a Gynecologic Oncology Group (GOG) 0262 trial was conducted for suboptimally operated patients. It is randomized phase III trial of every 3 weeks paclitaxel versus dose dense weekly paclitaxel in combination with carboplatin with or without concurrent and consolidation bevacizumab in the treatment of primary stage III or IV epithelial ovarian, peritoneal or fallopian tube cancer. The primary analysis demonstrated no significant difference in PFS in the patients who received the dose dense paclitaxel/carboplatin regimen compared to those who received the standard paclitaxel/carboplatin regimen (14.8 vs. 14.3 months, HR 0.97). However, there was a significant difference in PFS in the dose dense paclitaxel group (14.2 vs 10.3 months, HR 0.586 ) in a subset analysis of the small fraction of patients who did not receive bevacizumab.

The Multicentre Italian Trials in Ovarian Cancer (MITO-7) have launched in Italy, 2008. This randomized, multicenter, open-label, phase 3 trial assessed whether a weekly schedule of carboplatin (AUC 2) plus paclitaxel $\left(60 \mathrm{mg} / \mathrm{m}^{2}\right.$ ) is more effective than the same drug given every 3 weeks (AUC 6 of carboplatin plus $175 \mathrm{mg} / \mathrm{m}^{2}$ of paclitaxel) [5]. Unlike Japanese "NOVEL" trial, number of cases reporting grade 3-4 neutropenia was fewer in dose dense group than those allocated treatment every 3 weeks (42\% vs. 50\%). Another ongoing trial is ICON-8 trial which began in June, 2011 and whose estimated primary completion date is June, 2017. This trial is three-armed randomized trial comparing carboplatin AUC 5 plus $175 \mathrm{mg} / \mathrm{m}^{2}$ paclitaxel with carboplatin AUC 5 plus weekly $80 \mathrm{mg} / \mathrm{m}^{2}$ paclitaxel and with weekly carboplatin AUC 1.67 plus weekly $80 \mathrm{mg} / \mathrm{m}^{2}$ paclitaxel for stage IC to IV EOC.

\subsection{Route of Administration-IP Chemotherapy}

In addition to chemotherapeutic regimen, the route of administration chemoptherpeutic agents is important. Peritoneal seeding is the most frequent type of metastasis of advanced EOC, which results in a poor prognosis, with 5-year survival of around $<25 \%$ [6]. The potential advantage of IP chemotherapy is that administration of chemotherapeutic agents into intra-peritoneal cavity could increase the dose and exposure time of intra-abdominal cancer cells to anticancer drugs, with minimal systemic toxic effects [7]. IP chemotherapy has been attempted since 1980s. Multicenter randomized phase 3 trials and meta-analysis of IP chemotherapy have been performed and demonstrated that IP administrated regimens are superior to standard IV administrated regimens in patient with optimally debulked EOC. The representative study is GOG 172 trial; a phase III randomized 
Table 1. Clinical trials of currently accruing in ovarian cancer.

Clinical trial
BIBF 1120 in bevacizumab resistant,
persistent, or recurrent EOC
Veliparib monotherapy for relapsed
EOC with BRCA mutation
(Veli-BRCA)
Feasibility and clinical activity of
initial IP catumaxomab followed by
chemotherapy in patients with
recurrent EOC
Intraperitoneal therapy for ovarian
cancer with carboplatin trial (iPocc)

First-line intraperitoneal cisplatin and etoposide chemotherapy for ovarian cancer (AICE)

GANNET53: ganetespib in metastatic, p53-mutant, Platinum-resistant EOC

A study of rucaparib in patients with platinum-sensitive, relapsed, high-grade epithelial ovarian, fallopian tube, or primary peritoneal cancer (ARIEL2)

Bevacizumab and trabectedin $+/-$ carboplatin in advanced EOC

A study of pertuzumab in combination with standard chemotherapy in women with recurrent platinum-resistant EOC and Low HER3 mRNA expression

Bevacizumab beyond progression in platinum sensitive ovarian cancer

Phase II study of KPT-330 (selinexor) in female patients with advanced gynaecologic malignancies (SIGN)

Temsirolimus, carboplatin, and paclitaxel as first-line therapy in treating patients with newly diagnosed stage III-IV clear cell ovarian cancer

A randomized study of DNIB0600A in comparison with pegylated liposomal doxorubicin in patients with platinum-resistant ovarian cancer

Phase

2 BIBF 1120200 mg bid

1, 2 Veliparib $300 \mathrm{mg}$ bid daily

Catumaxomab 10, 20, 50,

$2150 \mu \mathrm{g}$ IP infusion on day $0,3,7,10$

IV paclitaxel: $80 \mathrm{mg} / \mathrm{m}^{2}$, on day $1,8,15$

2, 3 IP carboplatin: AUC $=6$ on day 1

IP: cisplatin $50 \mathrm{mg} / \mathrm{m}^{2}$, etoposide $100 \mathrm{mg} / \mathrm{m}^{2}$, weekly, 4 cycles

2 Followed IV: paclitaxel 175 $\mathrm{mg} / \mathrm{m}^{2}$ plus carboplatin

AUC 5 or docetaxel 60 -

$75 \mathrm{mg} / \mathrm{m}^{2}$ plus carboplatin

AUC 5 after 14 days

IV paclitaxel, $80 \mathrm{mg} / \mathrm{m}^{2}$

1, 2 Ganetespib on day 1, 8, 15 per 28-days cycle

2 Rucaparib twice a day

Bevacizumab (15 mg/kg)

2 followed by trabectedin $\left(1.1 \mathrm{mg} / \mathrm{m}^{2}\right)$ every 21 days

IV pertuzumab 840

$3 \mathrm{mg}$ followed by $420 \mathrm{mg}$ every 3 weeks with standard chemotherapy

Chemotherapy (pegylated

3 liposomal doxorubicin, gemcitabine, paclitaxel) and bevacizumab

2 Oral KPT-330 at $50 \mathrm{mg} / \mathrm{m}^{2}$ twice weekly

Paclitaxel and carboplatin chemotherapy plus

2 temsirolimus IV on days 1 and 8.

Consolidation: temsirolimus

IV on days $1,8,15$

2 DNIB0600A 2.4 mg/kg IV

every three weeks
Trial population

Recurrent or persistent EOC, progression or less than 6 months bevacizumab including treatment-free interval

Histologically confirmed EOC, with germline BRCA1/2 mutations

Platinum resistance or partially platinum sensitive disease

Response

rate

Recurrent ovarian cancer

Feasibility

Stage II-IV EOC, scheduled to undergo

laparotomy

(both optimal and suboptimal patients

will be eligible)

PFS

Stage IIIc or IV, expect for lymph node

12-month

metastasis alone, EOC

disease non-

Optimal cytoreductive surgery, with a residual progression

disease $\leq 1 \mathrm{~cm}$

rate

High-grade serous, high-grade endometrioid, or undifferentiated EOC. Primary platinum-resistant disease

High-grade (serous or endometrioid histology) EOC

Relapsed/progressive disease as confirmed by CT scan

Received $\geq 1$ prior platinum-based treatment regimen

EOC with PFS between 6 - 12 months

PFS at

6-months

Platinum-resistant or refractory EOC, with low HER3 mRNA expression

Safety, PFS

EOC with recurrence or progression at least 6 months after the last chemotherapy including bevacizumab

PFS

EOC: platinum refractory and platinum

resistant patients

Response

rate

Clear cell ovarian cancer at least 50\% clear cell histomorphology

Activity, PFS

Progressed or relapsed EOC during or within 6 months after the most recent treatment with a platinum-containing chemotherapy regimen and for whom PLD is appropriate therapy 


\section{Continued}

Pazopanib hydrochloride, paclitaxel, and carboplatin in treating patients with refractory or resistant EOC

Trametinib in treating patients with recurrent or progressive low-grade ovarian cancer

Pazopanib and weekly topotecan in patients recurrent ovarian cancer (TOPAZ)

A randomized study of safety and efficacy of pazopanib and gemcitabine in persistent or relapsed ovarian cancer
Pazopanib in combination

1, 2 with paclitaxel and

carboplatin chemotherapy

2, 3 Trametinib PO QD on days 1 - 28, every 28 days

Topotecan weekly 3 - 4

$1,2 \mathrm{mg} / \mathrm{m}^{2}$, pazopanib 400 ,

$600,800 \mathrm{mg}$ according to dose level

Gemcitabine $1000 \mathrm{mg} / \mathrm{m}^{2}$

2 on days 1,8 up to 6 cycles.

Pazopanib 800 mg PO

daily on days $1-21$
Platinum-refractory or resistant EOC

PFS

Low-grade serous ovarian carcinoma that

recur as low-grade serous carcinoma

PFS

Platinum resistant or refractory or

intermediate platinum-sensitive (recurrence

within 12 months after a platinum-based

primary therapy) EOC disease

Relapsed ovarian cancer previously with

carboplatin, cisplatin or another

PFS

trial of intravenous paclitaxel and cisplatin versus intravenous paclitaxel, intraperitonealcisplatin and intraperitoneal paclitaxel in patients with optimal stage III epithelial ovarian carcinoma or primary peritoneal carcinoma [8]. In this trial, IV paclitaxel plus cisplatin administration regimen was compared to IV paclitaxel plus IP cisplatin and paclitaxel administration regimen. The patients with stage 3 ovarian carcinoma or primary peritoneal carcinoma with no residual mass greater than $1.0 \mathrm{~cm}$ were randomly assigned to receive IV paclitaxel (135 $\mathrm{mg} / \mathrm{m}^{2}$ ) over a 24-hour period followed by either $75 \mathrm{mg} / \mathrm{m}^{2}$ of IV cisplatin on day 2 (IV-therapy group) or 100 $\mathrm{mg}$ of IP cisplatin per square meter on day 2 and $60 \mathrm{mg}$ of IP paclitaxel per square meter on day 8 (IP-therapy group). Treatment was given every three weeks for six cycles. It is reported that the adverse events were more common in the IP-therapy group than in the IV-therapy group $(\mathrm{p} \leq 0.001)$ and only 42 percent of the patients in the IP-therapy group completed six cycles of the assigned therapy. However, the median duration of progression-free survival (PFS) in the IV-therapy group and IP-therapy group were 18.3 and 23.8 months, respectively ( $\mathrm{p}=0.05$ by the log-rank test). And the median duration of OS in the IV-therapy group and IP-therapy group was 49.7 and 65.6 months, respectively $(p=0.03)$. Based on these studies, The National Comprehensive Cancer Network guidelines now recommend IP chemotherapy for patients with optimal cytoreductive surgery [9]. The recommended IP chemotherapy regimen is paclitaxel, $135 \mathrm{mg} / \mathrm{m}^{2}$ continuous infusion over 3 to 24 hours on day 1. Cisplatin 75 to $100 \mathrm{mg} / \mathrm{m}^{2}$ IP on day 2 after IV paclitaxel, paclitaxel $60 \mathrm{mg} / \mathrm{m}^{2}$ IP on day 8 and repeat every 3weeks times 6 cycles [9]. However, adjuvant IP chemotherapy has not been widely accepted and underused despite its survival benefits. Possible reasons are toxicities and inconvenience of IP therapy. IP chemotherapy has known to cause more gastrointestinal toxicity, pain, fever and infection than IV chemotherapy [10]. And there is additional risk of catheter-related complication [11]. In GOG 172 trial, only $42 \%$ of patients completed all 6 cycles of IP chemotherapy due to these toxicities and catheter related complications [8]. Though only 42\% of women were able to complete all 6 treatment cycles in GOG 172 trial, this percentage has been improved in the major cancer centers. Modification of regimen enables improved compliance to IP chemotherapy, and various chemotherapeutic agents and doses were tested to find optimal regimens. Using a lower IP cisplatin dose of $75 \mathrm{mg} / \mathrm{m}^{2}$ or splitting the dose may help to decrease toxicity [12]. Though it has not been proven to be equivalent to a 24-hour infusion, 3-hour infusion of paclitaxel has been reported to be more convenient with less toxicity profile [13] [14]. Based on inspiring result of dose dense chemotherapy, a randomized phase 2/3 trial is currently underway comparing IV dose dense paclitaxel plus IV carboplatin every 3 weeks to IV dose dense paclitaxel plus IP carboplatin every 3 weeks.

Pharmacokinetics after IP administration are important and are affected by a variety of biophysical parameters, including molecular weight, electric charge of drugs, temperature, $\mathrm{pH}$, and other conditions of the solution [7]. Some clinicians use hyper-thermic condition (HIPEC) to promote absorption of agents from the surface of peritoneal tumors, with minimal systemic toxic effects. However, to date, there is no prospective randomized trial and HIPEC is considered to be associated with high morbidity and mortality. Instead of conventional drug, nanodrugs are considered. Nanodrugs are significantly smaller than conventional drugs and are effectively accumulated in tumor tissue with enhanced permeability and retention [15]. Though there is no study with EOC, this nanodrug demonstrated antitumor effect in peritoneal metastatic cancer of esophageal cancer, gastric cancer and breast cancer [7]. 
Another new approach in IP chemotherapy is adoption of target agents [7]. The representative agents are catumaxomab and bevacizumab. Catumaxomab is a trifunctional monoclonal antibody and it binds to human EpCAM-positive cancer cells and activating a complex antitumor immune reaction such as antibody-dependent cellular cytotoxicity, phagocytosis and T-cell mediated cytotoxicity [16]. A randomized phase 2/3 trial of catumaxomab in patients with malignant ascites due to epithelial cancer, including ovarian, gastric, breast, pancreatic, colorectal, and endometrial cancers had been conducted in 2012. In this study, 258 patients with malignant ascites were randomized and treatment with catumaxomab enabled those patients to benefit from better quality of life for prolonged survival period [17]. Another possible agent is bevacizumab. Bevacizumabhas shown significant efficacy in combination with chemotherapy. However there is no clinical trial addressing the use of bevacizumab by IP administration.

\subsection{Targeted Therapies}

Despite various efforts to overcome recurrence of EOC, drug resistance is major problem and leading the highest mortality of disease entity to recurrent disease. For patients with platinum refractory and resistant disease, conventional cytotoxic agents showed limited responses and clinical insignificant survival gains despite therapy related toxicities. Targeted agents hold the promise of greater selectivity with lower toxicity than conventional cytotoxic chemotherapy. The most important advanced included the introduction of anti-angiogenic therapy and the assessment of PARP inhibitors.

\subsection{Anti-Angiogenic Therapy-Bevacizumab}

Angiogenesis is essential for tumor invasion and metastasis, and is required for tumor growth beyond $1-2 \mathrm{~mm}$ [18] [19]. The vascular endothelial growth factor (VEGF) pathway is the most widely studied angiogenic pathway in carcinogenesis. The bevacizumab was the first anti-angiogenesis agent to be advanced in to the phase 3 randomized trial for advanced EOC. Recently, Gynecologic Oncology Groupconducted three-arm phase 3 randomized controlled trial, (GOG-0218 trial) to assessed bevacizumab combined with carboplatin/paclitaxel in the upfront setting compared to carboplatin/paclitaxel alone [20]. In the first experimental arm, patients were treated with concurrent bevacizumab (15 mg/kg) followed by placebo maintenance (bevacizumab-initiation), while in the second experimental arm, patients received concurrent and maintenance bevacizumab every 21 days for up to 16 doses (bevacizumab-throughout). A total of 1873 women with previously untreated, advanced stage, epithelial ovarian, primary peritoneal and fallopian tube cancer were enrolled at 336 institutions in 4 countries. The median PFS was significantly prolonged in patients receiving prolonged bevacizumab-throughout arm when compared to chemotherapy alone (14.1 months vs. 10.3 months, $\mathrm{p}<0.0001)$. Relative to control, the hazard ratio (HR) for progression or death was 0.717 (95\% CI 0.625 to 0.824; p < 0.001) with bevacizumab throughout. However PFS was not significantly increased in patients who did not receive maintenance bevacizumab versus chemotherapy alone and no significant difference in OS among the three groups was identified. The rates of other adverse events including gastrointestinal fistula/perforation, proteinuria ( $\geq$ grade 3 ), neutropenia, thromboembolism, and wound disruptions were similar among the 3 treatment arms. Another phase 3 randomized controlled trial is ICON (International Cooperative Group for Ovarian Neoplasia)-7 trial. This trial is a twoarmed trial comparing carboplatin plus paclitaxel (six cycles) against carboplatin plus paclitaxel with bevacizumab every 3 weeks for 6 cycles, followed by 12 cycles of maintenance bevacizumab. The dose of bevacizumab $(7.5 \mathrm{mg} / \mathrm{kg}$ ) was half of that used in former (GOG 218) trial [21]. The PFS was significantly improved in the bevacizumab arm relative to control (19 vs. 17.3 months; HR 0.81, 95\% CI $0.7-0.94, p=0.0041$ ). In patients at high risk of recurrence (stage 4, stage 3 with residual disease less than $1 \mathrm{~cm}$ ), the benefits of bevacizumab were magnified, with a 5.4 month improvement in PFS. Mature OS data for ICON-7 was recently presented at the 2013 ESMO Annual Meeting in Amsterdam [22]. There was no significant difference in OS between control and the bevacizumab containing arm (58.6 vs. 58.0 months respectively, HR 0.99, p $=0.85$ ). Interestingly, the beneficial effects of bevacizumab in the subset of patients at high risk for progression (stage 4, stage 3 with residual disease less than $1 \mathrm{~cm}$ ) continued, with bevacizumab treatment resulting in a 9.4 month improvement in median OS (30.3 vs. 39.7 months; $p=0.0072$ ).

Bevacizumab therapy is mentioned as primary chemotherapy/primary adjuvant therapy regimens for stage II-IV EOC. However this therapy is belonged to category 3 (based upon any level of evidence, there is major NCCN disagreement that the intervention is appropriate). Because data from these two studies have not shown a 
statistically significant increase in OS and/or improved quality of life.

Bevacizumab also is tested in recurrent EOC. The OCEANS; a randomized, double blind, placebo-controlled phase III trial of chemotherapy with or without bevacizumab in patients with platinum-sensitive recurrent epithelial ovarian, primary peritoneal, or fallopian tube cancer; showed the efficacy and safety of bevacizumab in the treatment of patients with recurrent, platinum sensitive EOC [23]. Overall, 484 platinum sensitive (disease progression $\geq 6$ months after completion of front-line platinum based chemotherapy) patients were enrolled. These were randomly assigned to carboplatin/gemcitabine + bevacizumab vs. carboplatin/gemcitabine + placebo for 6 to 10 cycles. Bevacizumab or placebo was then continued until disease progression. Median PFS for the bevacizumab arm was superior to that for the control arm, 12.4 vs. 8.4 months, respectively (HR 0.484 , 95\% CI 0.388 - 0.605; $\mathrm{p}<0.0001$ ). Addition of bevacizumab significantly improved the objective response rate (ORR) (78.5\% vs. $57.4 \%$; $\mathrm{p}=0.0001$ ), with the majority being partial responses. The OS data is immature yet.

In patients with platinum resistant recurrent EOC, the AURELIA: a randomized phase III tiral evaluating bevacizumab plus chemoptherapy for platinum-resistant recurrent ovarian cancer; was conducted [24]. In this study, platinum resistant (disease progressed $<6$ months after completing platinum based therapy) patient were randomly assigned to single agent chemotherapy alone or with bevacizumab $(10 \mathrm{mg} / \mathrm{kg}$ every 2 weeks or 15 $\mathrm{mg} / \mathrm{kg}$ every 3 weeks). The PFS hazard ratio (HR) after PFS events in 301 of 361 patients was 0.48 (95\% CI, 0.38 to 0.60 , p < 0.001). Median PFS was 3.4 months with chemotherapy alone versus 6.7 months with bevacizumab-containing therapy. ORR was $11.8 \%$ versus $27.3 \%$, respectively $(\mathrm{p}=0.001)$. Although addition of bevacizumab treatment showed longer PFS and higher ORR, there was no significant difference in OS.

\subsection{Other Anti-Angiogenesis Agents}

Besides bevacizumab, other anti-angiogenetic agents, including pazopanib, cediranib and trebananib, are under clinical trials. The Arbeitsgemeinschaft Gynaekologische Onkologie (AGO)-OVAR 16 study is phase 3 randomized trial of maintenance daily pazopanib vs. placebo following primary platinum-based combination chemotherapy. Pazopanib is a tyrosine kinase inhibitor showing anti-angiogenic effect by inhibiting VEFGR, PDGFR and c-Kit signaling. In this study, pazopanib maintenance is associated with a significantly increased median PFS (17.9 months vs 12.3 months; HR 0.766, $p=0.0021)$ [25]. OS data is immature yet.

Another tyrosine kinase inhibitor, Cediranib, also showed activity in recurrent EOC as single agent [26]. In the ICON-6 trial, randomized, double blind, phase 3 trial in platinum sensitive recurrent EOC, cediranib maintenance showed significant improvement in PFS in the relative to control (12.5 vs. 9.4 months; HR 0.57, 95\% CI $0.45-0.74, \mathrm{p}=0.00001$ ). Additionally, a 2.7 month improvement in OS was identified in the cediranib plus platinum based chemotherapy followed by maintenance cediranib arm. ICON-6 is the first trial to demonstrate a significant improvement in OS anti-angiogenic agent. Other antiangiogenic agents included trebananib and nintedanib. Trebananib (AMG 386) targets the angiopoietin axis and inhibit angiogenic pathway. Inn TRINOVA-1: a phase 3 randomized, double blind trial, weekly paclitaxel in combination with trebananib $(15 \mathrm{mg} / \mathrm{kg})$ given weekly or placebo in women were compared in recurrent EOC [27]. There was a significant improvement in PFS in the trebananib arm relative to control (7.2 vs. 5.4 months; HR 0.66, 95\% CI $0.57-0.77$, p < 0.001). OS data is immature. Major adverse events were edema, ascites and pleural effusions though common toxicities of VEGF were not common. TRINOVA-3: A prospective, phase 3, randomized, double blind placebo controlled trial, is evaluating in up front treatment of advanced EOC treatment with paclitaxel and carboplatin. Nintedanib (Vargatef) is an angiokinase inhibitor against VEGFR, fibroblast growth factor receptor and platelet derived growth factor receptor. In phase 3 randomized placebo-controlled trial (AGO-OVAR12/LUME-Ovar 1), administering nintedanib with paclitaxel and carboplatin significantly increasing PFS (HR 0.84; 95\% CI 0.72 - 0.98 ; $\mathrm{p}=0.0239)$ in up front therapy [28].

\subsection{PARP Inhibitors-Olaparib}

PARP inhibitors are the first biologic agents to be used to treat EOC based upon pre-selection characteristics of the patient's cancer [29]. PARP inhibitors are most active in cancers with defects or aberrations in DNA repair. As DNA repair defects present in about half of high grade serous cancers (HGSC), PARP inhibitor has been of particular interest in this subset of patients [30].

Olaparib (AZD2281) is most actively investigated agent in EOC. As a single agent, olaparib has demonstrated anti-cancer activity in germline BRCA-mutated recurrent germline BRCA mutated EOC [31]. In another phase 2 
study, single-agent olaparib (400 mg BID dose) demonstrated efficacy in both germline BRCA as well as sporadic EOC without a known BRCA mutation [32]. Responses seen in the non-BRCA-associated cancers were associated with platinum sensitivity. The response rate was $50 \%$ in patients with platinum sensitive cancer while only $4 \%$ patients with platinum resistant and non-BRCA-associated cancer had a response. Several randomized phase 2 studies testing olaparib in recurrent EOC have been conducted against pegylated liposomal doxorubicin (PLD) for treatment of recurrent EOC [33]. In this study, recurrent EOC patients with known germline BRCA1 or BRCA2 mutation were randomized 1:1:1 to PLD $50 \mathrm{mg} / \mathrm{m}^{2}$ IV every 4 weeks, olaparib capsules $200 \mathrm{mg}$ BID continuously or olaparib capsules $400 \mathrm{mg}$ BID continuously. Though this was a small study of only 97 patients, this study demonstrated that a PARP inhibitor has activity comparable to PLD in recurrent BRCA-associated EOC and that the $200 \mathrm{mg}$ BID dose of olaparib has comparable activity to $400 \mathrm{mg}$ BID. As maintenance therapy, Ledermann et al. tested olaparib for platinum sensitive recurrent high grade serous carcinoma (HGSC) in a double-blinded study against placebo [34]. This study included both germline BRCA-associated and sporadic HGSC. Following platinum-based chemotherapy, 265 patients were randomized to placebo or olaparib $400 \mathrm{mg}$ BID daily. The PFS was 8.4 months in olaparib maintenance group while 4.8 months in placebo group. (HR 0.35, 95\% CI $0.25-0.49, \mathrm{p}<0.00001$ ) and median PFS for the olaparib group was 11.2 months compared to 4.3 months for the placebo group (HR $=0.18,95 \%$ CI $0.11-0.31, \mathrm{p}<0.00001)$. Based on these Phase 2 trials, phase 3 studies testing olaparib versus placebo as maintenance therapy for BRCA-associated EOC in both newly diagnosed and platinum-sensitive recurrent settings are on-going.

Combinations of PARP inhibitors with conventional chemotherapy agents have been evaluated. Because myelosupression is common in both treatments, use of full dose chemotherapy is difficult in combination setting. The dose-limiting toxicity (DLT) was myelosuppression in a phase 1 studies of olaparib combining with carboplatin or paclitaxel. Other toxicities included neutropenia, thrombocytopenia, anemia, fatigue and carboplatin hypersensitivity [29].

\subsection{Other PARP Inhibitors}

The other PARP inhibitors including veliparib, niraparib, rucaparib and BMN 673 [29]. Veliparib inhibits both PARP-1 and PARP-2 and has undergone testing as a single agents and combination with other chemotherapy agents. Phase 1 studies have been performed using veliparib in combination with topotecan, irinotecan, doxorubicin and cyclophosphamide. In the phase 1 study of veliparib (40 mg daily) and oral cyclophosphamide (50 $\mathrm{mg}$ once per day for 21 days), seven of thirty five patients with BRCA mutations had partial responses [35]. In phase 2 trial of single-agent veliparib in recurrent BRCA-related EOC, preliminary results as 8 of 31 patients experienced a PR [36]. Myelosupression was major toxicities as olaparib.

Niraparib (MK4827), a selective PARP-1 and PARP-2 inhibitor, has been discovered in 2009 [37]. In phase 1 trial in BRCA mutation carriers and patients with sporadic BRCA cancer, eight (40\%, 95\% CI 19 - 64) of twenty BRCA1 or BRCA2 mutation carriers with EOC had RECIST partial responses [38]. Antitumor activity was also reported in sporadic high-grade serous ovarian cancer. Myelosupression, fatigue and pneumonitis were doselimiting toxicities. Currently, a phase 3 study of niraparib versus placebo as maintenance therapy in patients with platinum sensitive, recurrent EOC, is accruing patients. Another PARP-1 and PARP-2 oral inhibitor, rucaparib (CO338 and AGO14699) has demonstrated anti-cancer responses for both platinum resistant and sensitive EOC patients in phase 1 study [39]. Rucaparib is currently undergoing further phase 2 and 3 trials in patients with HGSC.

\section{Combination Strategies}

In addition to, as single agents, novel target agents were tried as combination manner with other cytotoxic agents or target agents. The OCTAVIA: a phase II study evaluated front line bevacizumab plus weekly paclitaxel and tri-weekly carboplatin [40]. Patients received a median of six chemotherapy and seventeen bevacizumab cycles. For 189 enrolled patients, median PFS was 23.7 months (95\% CI, 19.8 - 26.4 months), 1-year PFS rate was 85.6\%, Response Evaluation Criteria in Solid Tumors (RECIST) response rate was $84.6 \%$ and median response duration was 14.7 months. Adverse events were no more common than in phase 3 bevacizumab trials. A combined with IP chemotherapy, GOG 252: phase III clinical trial of bevacizumab with IV versus IP chemotherapy in ovarian, fallopian tube and primary peritoneal carcinoma is underway to evaluated efficacy of bevacizumab for optimally cytoreduced patients. Evaluation of combination therapies between target agents is also underway. 
Phase 1 study of olaparib and combined with bevacizumab has conducted and olaparib $400 \mathrm{mg}$ BID with bevacizumab $10 \mathrm{mg} / \mathrm{kg}$ IV every 2 weeks were tolerable [41]. An ongoing randomized phase 2 study is testing the combination of cediranib plus olaparib in patients with platinum sensitive recurrent EOC. PARP inhibitors are also tried with inhibitors of other signaling pathways including PI3 kinase inhibitors. As the activation of the PI3K pathway occurs in as much as 70\% of all EOC, these strategies could increase the patients who may benefit from PARP inhibitors beyond BRCA-associated and/or HR-deficient cancers.

\section{Ascorbic Acid as Alternative Cancer Treatments}

Ascorbic acid has been known as antioxidant and helps prevent oxidative stress. Ascorbic acid has long been implicated in preventing and treating cancers [42]. Among the most enduring of alternative medical treatments, ascorbic acid is also one of the most popular. A survey published in 2010 evaluating the use of ascorbic acid among health care practitioners attending annual complementary medicine conferences found that $84 \%$ of complementary medicine practitioners reported using ascorbic acid for various conditions including cancer [43]. There are several mechanisms which intake of ascorbic acid may influence mortality among women with cancer. In preclinical studies, ascorbic acid can induce apoptosis in tumor cells and enhance antitumor effects of chemotherapy in vitro and in vivo [44] [45]. Ascorbic acid also exhibits antioxidant actions including neutralization of free radicals which may impact cancer progression [46]. And help protect and repair normal cells that are damaged by chemotherapy or radiation therapy, which can result in fewer or less severe adverse effects and improve compliance to cancer therapy [47]. Conversely, evidence from some laboratory studies and randomized trials suggested that use of antioxidant supplements, including ascorbic acid, during chemotherapy or radiation therapy may actually protect tumor cells and decrease the efficacy of treatment [48].

Although there are some reports of anti-cancer effect of ascorbic acid in animal study and in vivo study, in vivo and animal study, there are little studies in the literature regarding the effect of ascorbic acid to ovarian cancer patients. In sub-analysis of Women's Health Initiative (WHI) study, dietary intake and supplemental antioxidant nutrients including ascorbic acid were not associated with a reduction in ovarian cancer risk [49].

Recently, Harris, H.R., et al. conducted meta-analysis about the association between ascorbic acid supplement and survival in breast cancer patients. The summary RR for a $100 \mathrm{mg}$ per day increase in dietary ascorbic acid intake was 0.73 (95\% CI 0.59 - 0.89) for total mortality and 0.78 (95\% CI 0.64 - 0.94) for breast cancer-specific mortality [50]. This study has limitations as meta-analysis of observation studies. The dosage, formula and schedule of ascorbic acid differed between studies and the studies did not provide a rationale for them. Although this meta analysis could not control confounders that were not adjusted for in individual studies, they resulted post-diagnosis ascorbic acid supplement use may be associated with a reduced risk of mortality. Besides dietary supplement, high dose ascorbic acid treatment is also performed as alternative treatment. Owing to bioavailability and renal excretion, it is hard to get high concentration by current ascorbic acid dietary formula. However by IV administration, studies suggest that higher levels of ascorbic acid may cause the death of cancer cells in the laboratory. The mechanism of high dose ascorbic acid is not fully understood, and anti-tumor activity via generation of hydrogen peroxide and metabolite have been proposed [51]-[53]. Current clinical evidence for effect of high dose ascorbic acid are inconsistent.

\section{Conclusion and Perspectives}

Dose dense chemotherapy and IP chemotherapy are an effective treatment with improved PFS in patients with advanced EOC. Current ongoing trials would be critical to determine optimal dose and administration route. However conventional cytotoxic agents showed limited responses for patients with platinum resistance. To overcome limitation of conventional cytotoxic agents, various targeting agents have been developed and are under-development. Anti-angiogenesis and PARP inhibition are the most promising with positive randomized trial results. These agents demonstrate improvements in PFS with tolerable adverse events compared to cytotoxic agents. As PARP inhibitor demonstrates response in limited patients with BRCA mutation or homologous recombination (HR) deficient tumor, to select these patients is another challenge. Currently there is neither prospectively validated biomarker of HR-deficient EOC nor predicts responsiveness to PARP inhibitors. Genomic studies of cancer and miRNA studies could be meaningful in this aspect. The Cancer Genome Atlas (TCGA) project divided EOC into four groups based on gene expression patterns and provided the basis for an integrated view of miRNA and mRNA expression [54]. Mutations including TP53, NF1, BRCA1, BRCA2, RB1 and 
CDK12 were evaluated and pathway analyses suggested that HR was defective in about half of the EOC analised. Though further research is required, miRNA levels are promising as a diagnostic tool and miRNAs are evaluated as predictor of chemotherapy response and prognostic marker [55]. With these efforts improved outcomes would be fulfilled with less toxicity and better cost effectiveness in EOC.

\section{References}

[1] Siegel, R., Naishadham, D. and Jemal, A. (2013) Cancer Statistics, 2013. CA: A Cancer Journal for Clinicians, 63, 11-30. http://dx.doi.org/10.3322/caac.21166

[2] Norton, L. (2001) Theoretical Concepts and the Emerging Role of Taxanes in Adjuvant Therapy. The Oncologist, 6, 30-35. http://dx.doi.org/10.1634/theoncologist.6-suppl_3-30

[3] Katsumata, N., Yasuda, M., Takahashi, F., Isonishi, S., Jobo, T., Aoki, D., et al. (2009) Dose-Dense Paclitaxel Once a Week in Combination with Carboplatin Every 3 Weeks for Advanced Ovarian Cancer: A Phase 3, Open-Label, Randomised Controlled Trial. Lancet, 374, 1331-1338. http://dx.doi.org/10.1016/S0140-6736(09)61157-0

[4] Katsumata, N., Yasuda, M., Isonishi, S., Takahashi, F., Michimae, H., Kimura, E., et al. (2013) Long-Term Results of Dose-Dense Paclitaxel and Carboplatin versus Conventional Paclitaxel and Carboplatin for Treatment of Advanced Epithelial Ovarian, Fallopian Tube, or Primary Peritoneal Cancer (JGOG 3016): A Randomised, Controlled, OpenLabel Trial. The Lancet Oncology, 14, 1020-1026. http://dx.doi.org/10.1016/S1470-2045(13)70363-2

[5] Pignata, S., Scambia, G., Katsaros, D., Gallo, C., Pujade-Lauraine, E., De Placido, S., et al. (2014) Carboplatin plus Paclitaxel Once a Week versus Every 3 Weeks in Patients with Advanced Ovarian Cancer (MITO-7): A Randomised, Multicentre, Open-Label, Phase 3 Trial. The Lancet Oncology, 15, 396-405. http://dx.doi.org/10.1016/S1470-2045(14)70049-X

[6] Ozols, R.F. (2005) Treatment Goals in Ovarian Cancer. International Journal of Gynecological Cancer: Official Journal of the International Gynecological Cancer Society, 15, 3-11. http://dx.doi.org/10.1111/j.1525-1438.2005.15351.x

[7] Kitayama, J. (2014) Intraperitoneal Chemotherapy against Peritoneal Carcinomatosis: Current Status and Future Perspective. Surgical Oncology, 23, 99-106. http://dx.doi.org/10.1016/j.suronc.2014.03.004

[8] Armstrong, D.K., Bundy, B., Wenzel, L., Huang, H.Q., Baergen, R., Lele, S., et al. (2006) Intraperitoneal Cisplatin and Paclitaxel in Ovarian Cancer. The New England Journal of Medicine, 354, 34-43. http://dx.doi.org/10.1056/NEJMoa052985

[9] Morgan Jr., R.J., Alvarez, R.D., Armstrong, D.K., Boston, B., Burger, R.A., Chen, L.M., et al. (2011) Epithelial Ovarian Cancer. Journal of the National Comprehensive Cancer Network, 9, 82-113.

[10] Welfare Aloha. Australian Cancer Incidence and Mortality (ACIM) Books.

[11] Jaaback, K., Johnson, N. and Lawrie, T.A. (2011) Intraperitoneal Chemotherapy for the Initial Management of Primary Epithelial Ovarian Cancer. The Cochrane Database of Systematic Reviews, 2011, Article ID: CD005340. http://dx.doi.org/10.1002/14651858.CD005340.pub3

[12] Markman, M. (2009) An Update on the Use of Intraperitoneal Chemotherapy in the Management of Ovarian Cancer. Cancer Journal, 15, 105-109. http://dx.doi.org/10.1097/PPO.0b013e31819e31f2

[13] Barlin, J.N., Dao, F., Zgheib, N.B., Ferguson, S.E., Sabbatini, P.J., Hensley, M.L., et al. (2012) Progression-Free and Overall Survival of a Modified Outpatient Regimen of Primary Intravenous/Intraperitoneal Paclitaxel and Intraperitoneal Cisplatin in Ovarian, Fallopian Tube and Primary Peritoneal Cancer. Gynecologic Oncology, 125, 621-624. http://dx.doi.org/10.1016/j.ygyno.2012.03.027

[14] Blinman, P., Gainford, C., Donoghoe, M., Martyn, J., Blomfield, P., Grant, P., et al. (2013) Feasibility, Acceptability and Preferences for Intraperitoneal Chemotherapy with Paclitaxel and Cisplatin after Optimal Debulking Surgery for Ovarian and Related Cancers: An ANZGOG Study. Journal of Gynecologic Oncology, 24, 359-366.

[15] Matsumura, Y. and Maeda, H. (1986) A New Concept for Macromolecular Therapeutics in Cancer Chemotherapy: Mechanism of Tumoritropic Accumulation of Proteins and the Antitumor Agent Smancs. Cancer Research, 46, 63876392.

[16] Bokemeyer, C. (2010) Catumaxomab-Trifunctional Anti-EpCAM Antibody Used to Treat Malignant Ascites. Expert Opinion on Biological Therapy, 10, 1259-1269. http://dx.doi.org/10.1517/14712598.2010.504706

[17] Wimberger, P., Gilet, H., Gonschior, A.K., Heiss, M.M., Moehler, M., Oskay-Oezcelik, G., et al. (2012) Deterioration in Quality of Life (QoL) in Patients with Malignant Ascites: Results from a Phase II/III Study Comparing Paracentesis plus Catumaxomab with Paracentesis Alone. Annals of Oncology: Official Journal of the European Society for Medical Oncology, 23, 1979-1985.

[18] Eskander, R.N. and Randall, L.M. (2011) Bevacizumab in the Treatment of Ovarian Cancer. Biologics: Targets \& Therapy, 5, 1-5. 
[19] Folkman, J. (1971) Tumor Angiogenesis: Therapeutic Implications. The New England Journal of Medicine, 285, 11821186. http://dx.doi.org/10.1056/NEJM197111182852108

[20] Burger, R.A., Brady, M.F., Bookman, M.A., Fleming, G.F., Monk, B.J., Huang, H., et al. (2011) Incorporation of Bevacizumab in the Primary Treatment of Ovarian Cancer. The New England Journal of Medicine, 365, 2473-2483. http://dx.doi.org/10.1056/NEJMoa1104390

[21] Perren, T.J., Swart, A.M., Pfisterer, J., Ledermann, J.A., Pujade-Lauraine, E., Kristensen, G., et al. (2011) A Phase 3 Trial of Bevacizumab in Ovarian Cancer. The New England Journal of Medicine, 365, 2484-2496. http://dx.doi.org/10.1056/NEJMoa1103799

[22] Wagner, U., Marth, C., Largillier, R., Kaern, J., Brown, C., Heywood, M., et al. (2012) Final Overall Survival Results of Phase III GCIG CALYPSO Trial of Pegylated Liposomal Doxorubicin and Carboplatin vs Paclitaxel and Carboplatin in Platinum-Sensitive Ovarian Cancer Patients. British Journal of Cancer, 107, 588-591. http://dx.doi.org/10.1038/bjc.2012.307

[23] Aghajanian, C., Blank, S.V., Goff, B.A., Judson, P.L., Teneriello, M.G., Husain, A., et al. (2012) OCEANS: A Randomized, Double-Blind, Placebo-Controlled Phase III Trial of Chemotherapy with or without Bevacizumab in Patients with Platinum-Sensitive Recurrent Epithelial Ovarian, Primary Peritoneal, or Fallopian Tube Cancer. Journal of Clinical Oncology: Official Journal of the American Society of Clinical Oncology, 30, 2039-2045.

[24] Pujade-Lauraine, E., Hilpert, F., Weber, B., Reuss, A., Poveda, A., Kristensen, G., et al. (2014) Bevacizumab Combined with Chemotherapy for Platinum-Resistant Recurrent Ovarian Cancer: The AURELIA Open-Label Randomized Phase III Trial. Journal of Clinical Oncology: Official Journal of the American Society of Clinical Oncology, Published Online.

[25] Du Bois, A., Floquet, A., Kim, J.W., Rau, J., Maria Del Campo, J., Friedlander, M., Pignata, S., et al. (2013) Randomized, Double-Blind, Phase III Trial of Pazopanib versus Placebo in Women Who Have Not Progressed after FirstLine Chemotherapy for Advanced Epithelial Ovarian, Fallopian Tube, or Primary Peritoneal Cancer (AEOC): Results of an International Intergroup Trial (AGO-OVAR16). Journal of Clinical Oncology: Official Journal of the American Society of Clinical Oncology, 31, Article ID: LBA5503.

[26] Matulonis, U.A., Berlin, S., Ivy, P., Tyburski, K., Krasner, C., Zarwan, C., et al. (2009) Cediranib, an Oral Inhibitor of Vascular Endothelial Growth Factor Receptor Kinases, Is an Active Drug in Recurrent Epithelial Ovarian, Fallopian Tube and Peritoneal Cancer. Journal of Clinical Oncology: Official Journal of the American Society of Clinical Oncology, 27, 5601-5606.

[27] Monk, B.J., Poveda, A., Vergote, I., Raspagliesi, F., Fujiwara, K., Bae, D.S. and Oaknin, A. (2013) A Phase III, Randomized, Double-Blind Trial of Weekly Paclitaxel plus the Angiopoietin 1 and 2 Inhibitor, Trebananib or Placebo in Women with Recurrent Ovarian Cancer: TRINOVA-1. European Journal of Cancer, 49.

[28] DuBois, A., Kristensen, G., Ray-Coquard, I., Reuss, A., Pignata, S., Colombo, N. and Denison, U. (2013) AGO-OVAR 12: A Randomized Placebo-Controlled GCIG/ENGOT-Intergroup Phase III Trial of Standard Frontline Chemotherapy +/- Nintedanib for Advanced Ovarian Cancer. International Journal of Gynecological Cancer: Official Journal of the International Gynecological Cancer Society, 23, Oral Abstract.

[29] Liu, J.F., Konstantinopoulos, P.A. and Matulonis, U.A. (2014) PARP Inhibitors in Ovarian Cancer: Current Status and Future Promise. Gynecologic Oncology, 133, 362-369. http://dx.doi.org/10.1016/j.ygyno.2014.02.039

[30] Weberpals, J.I., Clark-Knowles, K.V. and Vanderhyden, B.C. (2008) Sporadic Epithelial Ovarian Cancer: Clinical Relevance of BRCA1 Inhibition in the DNA Damage and Repair Pathway. Journal of Clinical Oncology: Official Journal of the American Society of Clinical Oncology, 26, 3259-3267.

[31] Audeh, M.W., Carmichael, J., Penson, R.T., Friedlander, M., Powell, B., Bell-McGuinn, K.M., et al. (2010) Oral Poly(ADP-ribose) Polymerase Inhibitor Olaparib in Patients with BRCA1 or BRCA2 Mutations and Recurrent Ovarian Cancer: A Proof-of-Concept Trial. The Lancet, 376, 245-251. http://dx.doi.org/10.1016/S0140-6736(10)60893-8

[32] Gelmon, K.A., Tischkowitz, M., Mackay, H., Swenerton, K., Robidoux, A., Tonkin, K., et al. (2011) Olaparib in Patients with Recurrent High-Grade Serous or Poorly Differentiated Ovarian Carcinoma or Triple-Negative Breast Cancer: A Phase 2, Multicentre, Open-Label, Non-Randomised Study. The Lancet Oncology, 12, 852-861. http://dx.doi.org/10.1016/S1470-2045(11)70214-5

[33] Kaye, S.B., Lubinski, J., Matulonis, U., Ang, J.E., Gourley, C., Karlan, B.Y., et al. (2012) Phase II, Open-Label, Randomized, Multicenter Study Comparing the Efficacy and Safety of Olaparib, a Poly(ADP-Ribose) Polymerase Inhibitor, and Pegylated Liposomal Doxorubicin in Patients with BRCA1 or BRCA2 Mutations and Recurrent Ovarian Cancer. Journal of Clinical Oncology: Official Journal of the American Society of Clinical Oncology, 30, 372-379.

[34] Ledermann, J., Harter, P., Gourley, C., Friedlander, M., Vergote, I., Rustin, G., et al. (2012) Olaparib Maintenance Therapy in Platinum-Sensitive Relapsed Ovarian Cancer. The New England Journal of Medicine, 366, 1382-1392. http://dx.doi.org/10.1056/NEJMoa1105535

[35] Kummar, S., Ji, J., Morgan, R., Lenz, H.J., Puhalla, S.L., Belani, C.P., et al. (2012) A Phase I Study of Veliparib in 
Combination with Metronomic Cyclophosphamide in Adults with Refractory Solid Tumors and Lymphomas. Clinical Cancer Research: An Official Journal of the American Association for Cancer Research, 18, 1726-1734.

[36] Coleman, R.L., Sill, M. and Aghajanian, C. (2013) A Phase II Evaluation of the Potent, Highly Selective PARP Inhibitor Veliparib in the Treatment of Persistent or Recurrent Epithelial Ovarian, Fallopian Tube, or Primary Peritoneal Cancer in Patients Who Carry a Germline BRCA1 or BRCA2 Mutation-A Gynecologic Oncology Group Study. Proceedings of Society of Gynecologic Oncology 45th Annual Meeting on Women's Cancer, Tampa, 22-25 March 2014, Abstract 136.

[37] Jones, P., Altamura, S., Boueres, J., Ferrigno, F., Fonsi, M., Giomini, C., et al. (2009) Discovery of 2-\{4-[(3S)-Piperidin3-yl]Phenyl $\}$-2H-Indazole-7-Carboxamide (MK-4827): A Novel Oral Poly(ADP-Ribose)Polymerase (PARP) Inhibitor Efficacious in BRCA-1 and -2 Mutant Tumors. Journal of Medicinal Chemistry, 52, 7170-7185. http://dx.doi.org/10.1021/jm901188v

[38] Sandhu, S.K., Schelman, W.R., Wilding, G., Moreno, V., Baird, R.D., Miranda, S., et al. (2013) The Poly(ADP-Ribose) Polymerase Inhibitor Niraparib (MK4827) in BRCA Mutation Carriers and Patients with Sporadic Cancer: A Phase 1 Dose-Escalation Trial. The Lancet Oncology, 14, 882-892. http://dx.doi.org/10.1016/S1470-2045(13)70240-7

[39] Kristeleit, R.B.H., LoRusso, P., Patel, M., Giordano, H. and Evans, J. (2013) Phase I Study of Continuous Oral Rucaparib: Analysis of Patient Subgroup with Ovarian/Peritoneal Cancer. International Journal of Gynecological Cancer, 23, S564.

[40] Gonzalez-Martin, A., Gladieff, L., Tholander, B., Stroyakovsky, D., Gore, M., Scambia, G., et al. (2013) Efficacy and Safety Results from OCTAVIA, a Single-Arm Phase II Study Evaluating Front-Line Bevacizumab, Carboplatin and Weekly Paclitaxel for Ovarian Cancer. European Journal of Cancer, 49, 3831-3838.

[41] Dean, E., Middleton, M.R., Pwint, T., Swaisland, H., Carmichael, J., Goodege-Kunwar, P., et al. (2012) Phase I Study to Assess the Safety and Tolerability of Olaparib in Combination with Bevacizumab in Patients with Advanced Solid Tumors. British Journal of Cancer, 106, 468-474. http://dx.doi.org/10.1038/bjc.2011.555

[42] Cameron, E. and Pauling, L. (1976) Supplemental Ascorbate in the Supportive Treatment of Cancer: Prolongation of Survival Times in Terminal Human Cancer. Proceedings of the National Academy of Sciences of the United States of America, 73, 3685-3689. http://dx.doi.org/10.1073/pnas.73.10.3685

[43] Padayatty, S.J., Sun, A.Y., Chen, Q., Espey, M.G., Drisko, J. and Levine, M. (2010) Vitamin C: Intravenous Use by Complementary and Alternative Medicine Practitioners and Adverse Effects. PLoS ONE, 5, e11414. http://dx.doi.org/10.1371/journal.pone.0011414

[44] Drisko, J.A., Chapman, J. and Hunter, V.J. (2003) The Use of Antioxidant Therapies during Chemotherapy. Gynecologic Oncology, 88, 434-439. http://dx.doi.org/10.1016/S0090-8258(02)00067-7

[45] Prasad, K.N., Cole, W.C., Kumar, B. and Che Prasad, K. (2002) Pros and Cons of Antioxidant Use during Radiation Therapy. Cancer Treatment Reviews, 28, 79-91. http://dx.doi.org/10.1053/ctrv.2002.0260

[46] Frei, B. (1994) Reactive Oxygen Species and Antioxidant Vitamins: Mechanisms of Action. The American Journal of Medicine, 97, 5S-13S.

[47] Nakayama, A., Alladin, K.P., Igbokwe, O. and White, J.D. (2011) Systematic Review: Generating Evidence-Based Guidelines on the Concurrent Use of Dietary Antioxidants and Chemotherapy or Radiotherapy. Cancer Investigation, 29, 655-667. http://dx.doi.org/10.3109/07357907.2011.626479

[48] Lawenda, B.D., Kelly, K.M., Ladas, E.J., Sagar, S.M., Vickers, A. and Blumberg, J.B. (2008) Should Supplemental Antioxidant Administration Be Avoided during Chemotherapy and Radiation Therapy? Journal of the National Cancer Institute, 100, 773-783. http://dx.doi.org/10.1093/jnci/djn148

[49] Thomson, C.A., Neuhouser, M.L., Shikany, J.M., Caan, B.J., Monk, B.J., Mossavar-Rahmani, Y., et al. (2008) The Role of Antioxidants and Vitamin A in Ovarian Cancer: Results from the Women's Health Initiative. Nutrition and Cancer, 60, 710-719.

[50] Harris, H.R., Orsini, N. and Wolk, A. (2014) Vitamin C and Survival among Women with Breast Cancer: A MetaAnalysis. European Journal of Cancer, 50, 1223-1231. http://dx.doi.org/10.1016/j.ejca.2014.02.013

[51] Vance, T.M., Su, J., Fontham, E.T., Koo, S.I. and Chun, O.K. (2013) Dietary Antioxidants and Prostate Cancer: A Review. Nutrition and Cancer, 65, 793-801. http://dx.doi.org/10.1080/01635581.2013.806672

[52] Mamede, A.C., Pires, A.S., Abrantes, A.M., Tavares, S.D., Goncalves, A.C., Casalta-Lopes, J.E., et al. (2012) Cytotoxicity of Ascorbic Acid in a Human Colorectal Adenocarcinoma Cell Line (WiDr): In Vitro and in Vivo Studies. Nutrition and Cancer, 64, 1049-1057. http://dx.doi.org/10.1080/01635581.2012.713539

[53] Chen, Q., Espey, M.G., Sun, A.Y., Lee, J.H., Krishna, M.C., Shacter, E., et al. (2007) Ascorbate in Pharmacologic Concentrations Selectively Generates Ascorbate Radical and Hydrogen Peroxide in Extracellular Fluid in Vivo. Proceedings of the National Academy of Sciences of the United States of America, 104, 8749-8754. http://dx.doi.org/10.1073/pnas.0702854104 
J. Seoung et al.

[54] Cancer Genome Atlas Research Network (2011) Integrated Genomic Analyses of Ovarian Carcinoma. Nature, 474, 609-615. http://dx.doi.org/10.1038/nature10166

[55] Davidson, B., Trope, C.G. and Reich, R. (2014) The Clinical and Diagnostic Role of microRNAs in Ovarian Carcinoma. Gynecologic Oncology, 133, 640-646. http://dx.doi.org/10.1016/j.ygyno.2014.03.575 
Scientific Research Publishing (SCIRP) is one of the largest Open Access journal publishers. It is currently publishing more than 200 open access, online, peer-reviewed journals covering a wide range of academic disciplines. SCIRP serves the worldwide academic communities and contributes to the progress and application of science with its publication.

Other selected journals from SCIRP are listed as below. Submit your manuscript to us via either submit@scirp.org or Online Submission Portal.
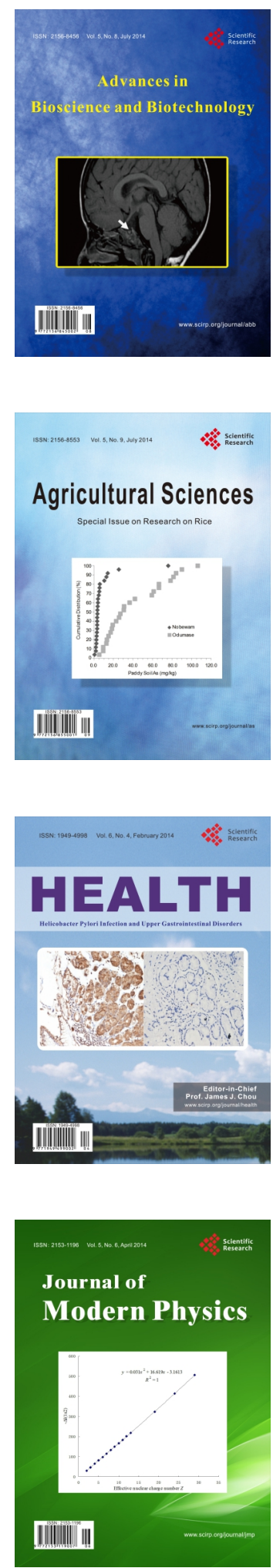
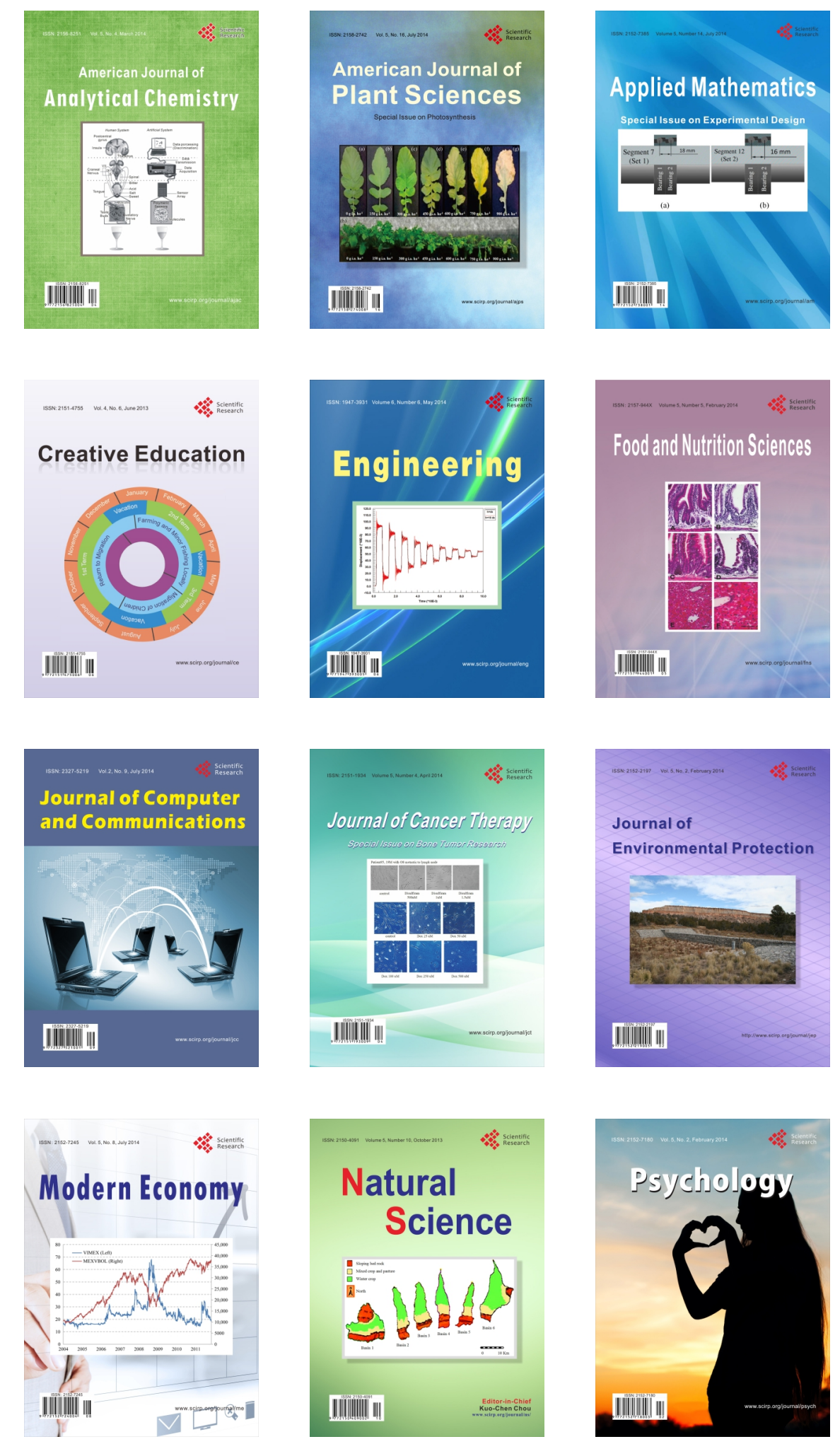